'Departamento de Nutrición y Dietética y Programa de Magister en Nutrición Humana Facultad de Farmacia, Universidad de Concepción Concepción, Chile.

2BHF Glasgow Cardiovascular Research Centre, Institute of Cardiovascular and Medical Sciences, University of Glasgow, Glasgow, United Kingdom ${ }^{3}$ Instituto de Farmacia, Facultad de Ciencias, Universidad Austral de Chile, Valdivia, Chile. "Instituto de Anatomía, Histología y Patología Facultad de Medicina, Universidad Austral de Chile, Valdivia, Chile.

'Escuela de Educación Física, Universidad San Sebastián, Concepción, Chile.

${ }^{6}$ Escuela de Kinesiología, Facultad de Salud Universidad Santo Tomás, Sede Valdivia. Chile. 7 Grupo calidad vida. Departamento Ciencias de la Educación, Facultad de Educación y Humanidades, Universidad del Biobío, Chillán, Chile.

${ }^{8}$ Departamento de Educación Física. Facultad de Educación. Universidad de Concepción. Concepción, Chile.

Nutricionista. MSc. Nutrición Humana. ${ }^{b}$ Nutricionista. MSc. Planificación en Alimentación y Nutrición.

'Nutricionista. MSc. Ciencias de la Educación. ${ }^{d}$ Bioquímica. MSc. Nutrición y Dietética. eProfesora de Biología y Química. MSc. Neurociencias y Salud Mental. Profesor de Educación Física, Doctor en Actividad física, Educación Física y Deporte. gProfesor de Educación Física, MSc. Educación en Salud y Bienestar Humano.

hProfesor de Educación Física, MSc Educación. Profesor de Educación Física, MSc Educación Física. jProfesor de Educación Física, Doctor en Ciencias Cardiovasculares y Biomédicas.

*FP, AML y ED contribuyeron igualmente y son consideradas primeras autoras compartidas. Recibido el 20 de marzo de 2017, aceptado el 5 julio de 2017

Correspondencia a: Dr. Carlos Celis-Morales BHF Glasgow Cardiovascular Research Centre, 126 University Avenue, Glasgow University Glasgow, United Kingdom, G12 8TA Teléfono: +(0)4401413304201 carlos.celis@glasgow.ac.uk

\section{Factores asociados al desarrollo de obesidad en Chile: resultados de la Encuesta Nacional de Salud 2009-2010}

\author{
FANNY PETERMANN 1,2,a*, ELIANA DURÁN ${ }^{1, b, *}$, \\ ANA MARÍA LABRAÑA ${ }^{1, \mathrm{c}, *}$, MARÍA ADELA MARTÍNEZ ${ }^{3, \mathrm{~d}}$, \\ ANA MARÍA LEIVA, ${ }^{4, e}$, ALEX GARRIDO-MÉNDEZ ${ }^{5, \mathrm{f}}$, \\ FELIPEPOBLETE-VALDERRAMA ${ }^{6, \mathrm{~g}}$, XIMENADÍAZ-MARTÍNEZ $^{7, \mathrm{~h}}$, \\ CARLOS SALAS ${ }^{8, i}$, CARLOS CELIS-MORALES $^{2, j}$
}

\section{Risk factors for obesity: analysis of the 2009-2010 Chilean health survey}

Background: Chile has one of the highest obesity rates in Latin America. However, the factors that could explain this high prevalence of obesity are unknown. Aim: To determine the main risk factors for the development of obesity in Chile. Material and Methods: We included 1,398 obese and 1,478 normal weight participants of the National Health Survey 2009-2010. The risk factors considered were socioeconomic variables, diet, physical activity, comorbidities and general wellbeing. Results: The main factors associated with a higher risk of being obese were age, high salt consumption and high alcohol intake. Education, income and living in rural settings were protective for women and risk factors for men. The probability of being obese increases in women that sleep less than seven hours per day or have a poor health. Sedentariness and lack of physical activity were risk factors among men. Conclusions: The identification of these risk factors may help to implement public health interventions to tackle obesity in Chile.

(Rev Med Chile 2017; 145: 716-722)

Key words: Body Mass Index; Obesity; Sedentary Lifestyle.

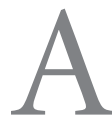
pesar de los esfuerzos realizados a nivel de salud pública, la obesidad ha aumentado en las últimas décadas, convirtiéndose en uno de los principales factores de riesgo de morbilidad y mortalidad $^{1-3}$. En el año 2014, más de 600 millones de adultos a nivel mundial presentaban esta enfermedad ${ }^{4}$. En Chile, la última Encuesta Nacional de Salud (ENS) 20092010, evidenció que su prevalencia alcanzó a $28 \%$ de la población mayor de 18 años, según índice de masa corporal (IMC $\geq 30 \mathrm{~kg} / \mathrm{m}^{2}$ ), siendo las mujeres quienes presentaron una mayor prevalencia ${ }^{5}$. Estos datos sitúan a Chile en la primera posición del ranking de obesidad en Latinoamérica ${ }^{6}$.

La evidencia sugiere que la obesidad se relaciona directamente con cambios en el estilo de vida $^{7,8}$ como la inactividad física, insuficientes horas de sueño, excesivo consumo de alcohol y el hábito de fumar, aunque la implicancia de estos dos últimos aún no está muy clara ${ }^{9}$. Además de la edad y el sexo, la genética, los factores fisiológicos, 
ambientales y socioeconómicos también han sido asociados con obesidad ${ }^{10,11}$.

A pesar de ser conocidos los principales factores de riesgo relacionados con esta patología, se desconoce cuáles de ellos podrían tener una mayor o menor implicancia en el desarrollo de ésta en nuestro país. Por lo anterior, el objetivo de este estudio fue determinar los principales factores de riesgo asociados al desarrollo de obesidad en Chile.

\section{Materiales y Métodos}

\section{Diseño del estudio}

La muestra seleccionada comprendió 2.876 participantes de la ENS 2009-2010, quienes presentaron un estado nutricional normal o de obesidad según IMC al momento de la medición ${ }^{5}$. La base de datos fue proporcionada por el Departamento de Epidemiología del Ministerio de Salud. La ENS 2009-2010 corresponde a un estudio de prevalencia realizado en hogares en una muestra nacional, probabilística, estratificada y multietápica de 5.412 personas mayores de 15 años con representatividad nacional, regional, $y$ área urbano/rural.

Los participantes firmaron un consentimiento informado y sus resultados les fueron devueltos con recomendaciones y derivación según correspondía ${ }^{5}$. El protocolo de estudio fue aprobado por el Comité de Ética de Investigación de la Escuela de Medicina de la Pontificia Universidad Católica de Chile.

\section{Mediciones antropométricas y metabólicas}

Las mediciones antropométricas fueron realizadas por personal entrenado y bajo protocolos descritos en extenso en la ENS ${ }^{5}$. Se clasificó el estado nutricional según IMC en base a las recomendaciones de la Organización Mundial de la Salud ${ }^{4}$. La obesidad central fue definida tras la medición del perímetro de cintura (PC) y en base a los siguientes puntos de corte: $\geq 83 \mathrm{~cm}$ para mujeres $\mathrm{y} \geq 88 \mathrm{~cm}$ para hombres 5 .

Las muestras de sangre, realizadas en ayuno, fueron obtenidas por una enfermera entrenada siguiendo protocolos estandarizados a nivel nacional ${ }^{5}$. Los marcadores metabólicos de glicemia, y presión arterial fueron medidos con métodos estandarizados y previamente descritos en la ENS junto a sus respectivos puntos de corte ${ }^{5}$.

\section{Clasificación de actividad física}

Los niveles de actividad física (AF), el tiempo destinado a las actividades de transporte, y las actividades de intensidad moderada o vigorosa de la población, fueron determinados con el cuestionario "Global Physical Activity Questionnaire" (GPAQ v2) ${ }^{12}$, el cual ha sido validado internacionalmente ${ }^{13} \mathrm{y}$ en población latina ${ }^{14}$. Los niveles de sedentarismo fueron determinados mediante el auto-reporte de tiempo destinado a actividades que involucren estar sentado o reclinado durante el tiempo libre o de trabajo. Los niveles de AF total fueron expresadas en METs (Metabolic Energy Equivalents) ${ }^{15}$. Se consideró como punto de corte para inactividad física un gasto energético menor a $600 \mathrm{METs} / \mathrm{minuto}_{\mathrm{semana}}{ }^{12,16}$.

\section{Estimación del índice de dieta saludable (IDS)}

La estimación del índice de dieta saludable (IDS) fue realizado en base a la metodología reportada por Dussaillant y cols. en el año $2015^{17}$. El IDS se calculó como la suma de los puntajes asignados a 4 grupos de alimentos: verduras, frutas, cereales integrales y pescado, quedando con un rango posible de puntaje entre 0 a 4 puntos (un puntaje alto refleja una alimentación saludable, mientras que un puntaje bajo refleja una alimentación no saludable).

Para determinar las variables de hábito tabáquico, se consideraron preguntas basadas en el instrumento mínimo de vigilancia de tabaquismo, utilizado por la Organización Panamericana de la Salud. Las variables demográficas como edad, sexo, zona geográfica de residencia (urbano/rural), nivel de escolaridad (básica $<8$ años, media 8 a 12 años, y educación superior $>12$ años) e ingreso económico (Bajo < 250.000; Medio 250.000 a 650.000; Alto $>650.000$ pesos chilenos) fueron recolectadas mediante el uso de cuestionarios validados en población nacional ${ }^{5}$. El tiempo promedio destinado a dormir en un día normal y la auto-percepción de salud fueron recolectadas mediante encuesta y han sido descritas anteriormente en la ENS 2009-2010.

\section{Análisis estadístico}

Variables de tipo continuas son presentadas como promedio y su respectiva desviación standard (DS), mientras que variables categóricas son presentadas como porcentaje (\%). Para establecer diferencias significativas entre variables 
de tipo continua, se utilizó la prueba de t-test para muestras independientes y $\chi^{2}$ para variables categóricas.

Para determinar los principales factores asociados al desarrollo de obesidad (IMC $\geq 30,0 \mathrm{~kg} /$ $\mathrm{m}^{2}$ ) se realizaron análisis de regresión logística ajustando los modelos por variables de tipo confundentes. Estos análisis fueron ajustados por edad, sexo, tabaquismo, educación e ingreso económico, debido a que estos factores se asocian tanto a la variable dependiente (obesidad) como también a las variables independientes (sociodemográficas, actividad física, salud y dieta). Los datos para estos análisis fueron presentados como Odds ratio (OR) y sus respectivo 95\% de intervalos de confianza (95\% IC). Para todos los análisis se utilizó el módulo de análisis de muestras complejas del programa STATA SE v14 y todos los resultados fueron estimados utilizando muestras expandidas según la ENS 2009-20105. El nivel de significancia fue definido como $\mathrm{p}<0,05$.

\section{Resultados}

En la Tabla 1 se presentan las características generales de la población normopeso y obesa. Personas clasificadas como obesas presentan una mayor edad, un menor nivel de escolaridad, y un mayor porcentaje de ellas habita en zonas rurales, son mujeres y/o exfumadores, en comparación a la población normopeso. En relación a los estilos de vida, las personas obesas reportaron un mayor consumo de sal y niveles de actividad física de intensidad moderada, pero a su vez, una mayor prevalencia de inactividad física, de diabetes mellitus 2 (DM2) e hipertensión arterial (HTA) junto a bajos niveles de horas destinadas a dormir $(<7 \mathrm{~h}$ al día), en comparación a personas normopeso.

Con respecto a los factores asociados a obesidad, se pudo determinar que las mujeres presentan una mayor probabilidad de ser obesas que los hombres (OR: 1,41 [1,20 a 1,66], p < 0,0001). Al estratificar los análisis según sexo, encontramos que en hombres la probabilidad de ser obesos es mayor en grupos etarios $\geq 25$ años en comparación al grupo más joven $<25$ años (Figura 1). Los hombres que viven en zonas urbanas, aquellos con niveles de escolaridad media o superior, y/o con ingreso económico medio o alto presentan una mayor probabilidad de ser obesos. En relación a los estilos de vida, los hombres exfumadores, los que reportaron niveles medio o alto de sedentarismo, los que son físicamente inactivos y/o aquellos que reportaron un consumo medio o alto de alcohol y sal, presentan una mayor probabilidad de ser obesos. En relación a factores asociados a salud, hombres que presentan DM2 y/o HTA también tienen una mayor probabilidad. Por otro lado, existe un factor protector en aquellos que duermen más de $9 \mathrm{~h}$ al día en comparación a aquellos que duermen entre 7 a 9 h como se ilustra en la Figura 1. No se observaron asociaciones significativas entre obesidad y reporte de estado de salud y bienestar, consumo de frutas y verduras e IDS.

En la Figura 2 se presentan los factores asociados a obesidad en mujeres. Tal como se observó en hombres, la probabilidad de ser obesas también aumenta en todos los grupos etarios $\geq 25$ años, así como también en exfumadoras, aquellas con DM2, HTA, las que reportaron un consumo medio o alto de sal y un consumo alto de alcohol. Sin embargo, la probabilidad de ser obesa es opuesta a lo observado en hombres para zona geográfica, educación y nivel de ingreso. Las mujeres que viven en zonas urbanas presentan un nivel de escolaridad media o superior, tienen altos niveles de ingreso, y/o presentaron un alto puntaje en el IDS, poseen una menor probabilidad de ser obesas. Sin embargo, las mujeres que reportaron un mal estado de salud y bienestar o aquellas que reportaron dormir menos de $7 \mathrm{~h}$ al día tienen una mayor probabilidad de desarrollar la patología. No se observaron asociaciones significativas entre obesidad y sedentarismo, inactividad física y consumo de frutas y verduras.

\section{Discusión}

Como se ha señalado anteriormente, son diversas las causas y los factores de riesgo asociados al desarrollo de obesidad. En Chile, una edad superior a 25 años, ser mujer, mantener un consumo medio o alto de sal y un consumo alto de alcohol se asocian a un mayor riesgo, lo cual coincide con datos encontrados en la literatura ${ }^{9,10,18,19}$. Con respecto a la asociación con otras enfermedades, en ambos sexos, la obesidad se relaciona con DM2 e HTA. Lanas y cols., determinaron que en América Latina la obesidad se relaciona directamente con 
Tabla 1. Características de la población según estado nutricional por IMC

\begin{tabular}{|c|c|c|c|}
\hline & Normal & Obeso & Valor $\mathbf{p}$ \\
\hline Total (n) & 1.478 & 1.398 & - \\
\hline Edad (años) & $40,5 \pm 20,2$ & $50,2 \pm 16,5$ & $<0,0001$ \\
\hline Sexo, \% Mujeres & 59,9 & 67,7 & $<0,0001$ \\
\hline $\begin{array}{l}\text { Zona geográfica (\%) } \\
\text { Urbano }\end{array}$ & 87,4 & 83,4 & 0,003 \\
\hline $\begin{array}{l}\text { Nivel educacional (\%) } \\
\text { Básica (<8 años) } \\
\text { Media (< } 12 \text { años) } \\
\text { Superior (estudios superiores) }\end{array}$ & $\begin{array}{l}20,2 \\
57,5 \\
22,3\end{array}$ & $\begin{array}{l}32,7 \\
53,6 \\
13,7\end{array}$ & $<0,0001$ \\
\hline $\begin{array}{l}\text { Ingreso económico (\%) } \\
\text { Bajo } \\
\text { Medio } \\
\text { Alto }\end{array}$ & $\begin{array}{l}55,0 \\
32,4 \\
12,6\end{array}$ & $\begin{array}{l}59,0 \\
31,8 \\
9,2\end{array}$ & 0,009 \\
\hline $\begin{array}{l}\text { Fumador (\%) } \\
\text { No-fumador } \\
\text { Exfumador } \\
\text { Fumador }\end{array}$ & $\begin{array}{l}42,9 \\
18,4 \\
38,7\end{array}$ & $\begin{array}{l}41,4 \\
28,1 \\
30,5\end{array}$ & $<0,0001$ \\
\hline Peso $(\mathrm{Kg})$ & $58,3 \pm 8,4$ & $85,9 \pm 13,2$ & $<0,0001$ \\
\hline Talla (m) & $1,61 \pm 0,09$ & $1,59 \pm 0,1$ & $<0,0001$ \\
\hline IMC $\left(\mathrm{Kg} / \mathrm{m}^{2}\right)$ & $22,4 \pm 2,0$ & $34,2 \pm 4,5$ & $<0,0001$ \\
\hline Circunferencia cintura (cm) & $86,2 \pm 8,8$ & $108,3 \pm 10,8$ & $<0,0001$ \\
\hline Obesidad central (\%) & 22,1 & 99,4 & $<0,0001$ \\
\hline Actividad física total (MET/h/semana) & $117,5 \pm 144,0$ & $117,1 \pm 144,0$ & 0,933 \\
\hline Actividad física transporte (min/día) & $52,6 \pm 91,0$ & $47,6 \pm 84,6$ & 0,135 \\
\hline Actividad física moderada (min/día) & $96,7 \pm 3,7$ & $107,9 \pm 4,1$ & 0,044 \\
\hline Actividad física vigorosa (min/día) & $51,2 \pm 3,2$ & $47,7 \pm 3,1$ & 0,430 \\
\hline Tiempo de sedentarismo (h/día) & $3,5 \pm 2,6$ & $3,4 \pm 2,6$ & 0,370 \\
\hline Inactividad física (\%) & 21,4 & 28,2 & $<0,0001$ \\
\hline Consumo de frutas y verduras (g/día) & $209,2 \pm 136,0$ & $220,7 \pm 137,0$ & 0,035 \\
\hline Consumo de frutas y verduras todos los días (\%) & 36,4 & 33,8 & 0,139 \\
\hline Consumo de alcohol (g/día) & $53,0 \pm 69,4$ & $62,0 \pm 125,1$ & 0,179 \\
\hline Consumo de sal (g/día) & $8,9 \pm 2,1$ & $10,2 \pm 2,4$ & $<0,0001$ \\
\hline Índice de dieta saludable (IDS) & $1,5 \pm 0,9$ & $1,5 \pm 0,9$ & 0,992 \\
\hline $\begin{array}{l}\text { Horas de sueño (\%) } \\
\text { Normal }(7-9 \mathrm{~h}) \\
\text { Bajo }(<7 \mathrm{~h}) \\
\text { Alto }(>9 \mathrm{~h})\end{array}$ & $\begin{array}{l}52,8 \\
25,1 \\
22,1\end{array}$ & $\begin{array}{l}50,5 \\
31,4 \\
18,1\end{array}$ & $<0,0001$ \\
\hline $\begin{array}{l}\text { Autopercepción de salud y bienestar (\%) } \\
\text { Mala } \\
\text { Regular } \\
\text { Buena }\end{array}$ & $\begin{array}{l}3,1 \\
28,4 \\
68,5\end{array}$ & $\begin{array}{l}3,6 \\
36,2 \\
60,2\end{array}$ & $<0,0001$ \\
\hline Diabetes mellitus tipo 2 (\%) & 5,8 & 17,5 & $<0,0001$ \\
\hline Hipertensión (\%) & 18,8 & 44,7 & $<0,0001$ \\
\hline
\end{tabular}

Datos presentados como media y su respectiva desviación estándar para variables continuas y cómo \% para variables categóricas. Valor $\mathrm{p}$ representa diferencias significativas entre normopeso y obesos, este valor fue estimado con t-test para variables continuas y $\chi^{2}$ para variables categóricas. 


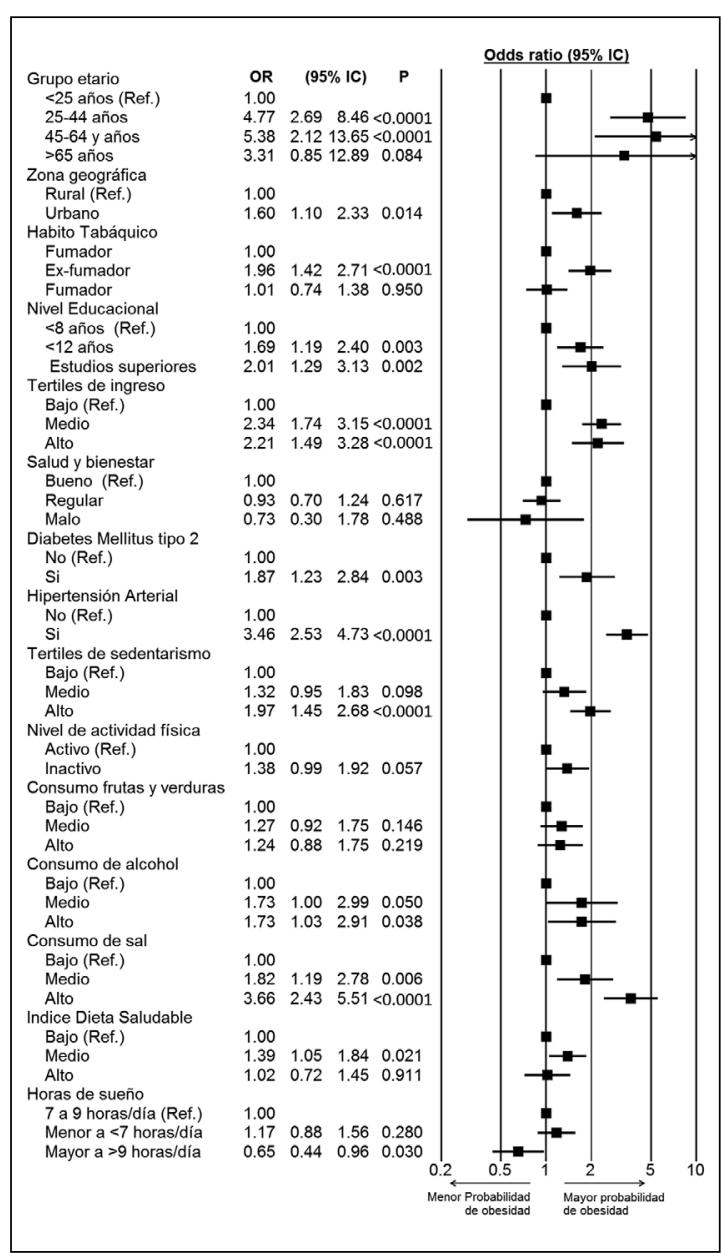

Figura 1. Factores de riesgo asociados a obesidad en hombres. Datos presentados como odds ratio y sus respectivos 95\% IC. Los análisis fueron ajustados por edad, zona geográfica, tabaquismo, y educación, excepto cuando estos fueron utilizados como factor de riesgo en los análisis. Odds ratio fue estimado mediante regresión logística, y asociaciones significativas fueron consideradas con un valor $p<0,05$.

DM2, HTA, dislipidemias y síndrome metabóli$\mathrm{Co}^{19}$. No obstante, los principales resultados de este estudio entregan evidencia que los factores de riesgo asociados a ser obeso difieren entre sexos. Nuestro estudio determinó que el nivel de escolaridad, ingreso económico y zona geográfica de residencia, presentan una relación opuesta en ambos sexos, considerándose factores protectores para mujeres y de riesgo para hombres. En el caso de los hombres, esto podría deberse al tiempo sedente que implica gran parte de los trabajos de

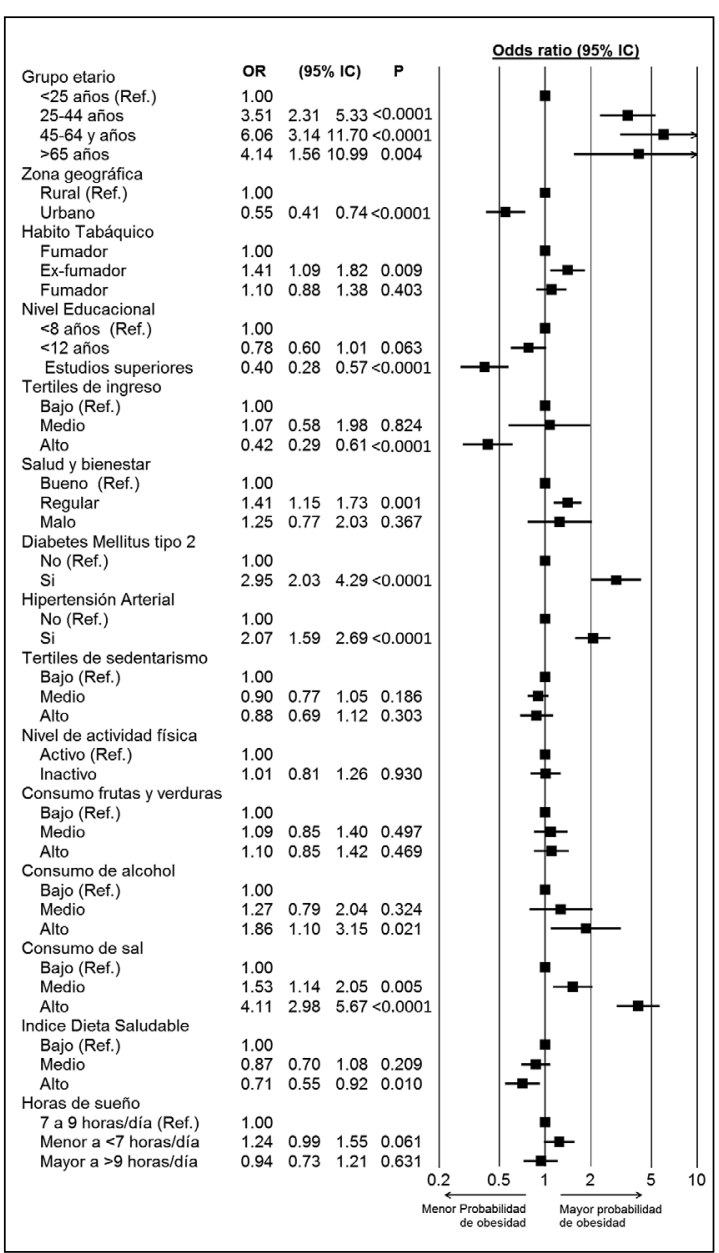

Figura 2. Factores de riesgo asociados a obesidad en mujeres. Datos presentados como odds ratio y sus respectivos $95 \%$ IC. Los análisis fueron ajustados por edad, zona geográfica, tabaquismo, y educación, excepto cuando estos fueron utilizados como factor de riesgo en los análisis. Odds ratio fue estimado mediante regresión logística, y asociaciones significativas fueron consideradas con un valor $p<0,05$.

altos ingresos en zonas urbanas; en el caso de las mujeres, podría estar asociado con una mayor inversión de recursos, esfuerzo y tiempo para mantener un peso adecuado con una alimentación equilibrada e hipocalórica ${ }^{18}$. La literatura frente a esto presenta datos contradictorios ${ }^{9,11,20}$.

Respecto a los estilos de vida, tanto hombres como mujeres exfumadores presentan mayor riesgo de desarrollar obesidad, situación que puede generarse al utilizar el tabaco como mecanismo de control de peso. Estos datos coinciden con 
los publicados por la literatura ${ }^{21,22}$ y por el grupo PREDIMERC, quienes identificaron que las mujeres fumadoras y aquellas con estudios universitarios presentan un factor protector frente a esta enfermedad $^{18}$. El estudio realizado por Pengpida y Peltzer en 31 países, también demostró que el consumo moderado o alto de alcohol se relaciona con obesidad, principalmente en hombres, y que la realización de actividad física diaria, semanal o en muchas ocasiones durante el mes, presentan un efecto protector ${ }^{10}$. Esto último coincide con los resultados de este estudio en donde el sedentarismo e inactividad física fueron asociados a una mayor probabilidad de ser obeso en hombres. Por otra parte, dormir más de $9 \mathrm{~h}$ al día se identificó como un factor protector en hombres y dormir menos de $7 \mathrm{~h}$ al día o tener un estado de salud y bienestar regular, se asoció a un mayor riesgo en mujeres. López-Sobale y cols., identificaron que en población española dormir sobre $7 \mathrm{~h}$ se considera como un factor protector 9 .

En relación a los hábitos alimentarios, presentar un IDS alto se asoció a una menor probabilidad de obesidad en mujeres, no así en hombres. No se encontró un factor protector relacionado al consumo de frutas y verduras, no obstante, otros grupos si lo han identificado ${ }^{10}$. Estas diferencias podrían explicarse debido a la medición auto-reportada que se realizó en relación al consumo de frutas y verduras, las cuales podrían estar sesgadas. Finalmente, respecto al consumo de sal, un grupo de científicos alemanes demostraron que las personas obesas son más sensibles al sabor y perciben más dulce, salado y amargo que las personas con estado nutricional normal ${ }^{23}$. Esto podría explicar el aumento del consumo de estos alimentos a medida que aumenta el IMC.

Considerando las últimas estadísticas en obesidad publicadas por la $\mathrm{OMS}^{6}$, que sitúan a Chile en la posición número uno del ranking de países con mayores niveles de obesidad en Latino América, los hallazgos de este estudio podrían tener importantes implicaciones en la definición de políticas públicas y en el diseño de intervenciones en Chile, ya que permitirían orientar a las entidades correspondientes respecto a cuáles son los grupos y características de las personas en el país que presentan una mayor probabilidad de ser obesas y en consecuencia tienen una mayor probabilidad de desarrollar enfermedades crónicas no transmisibles y un mayor riesgo de muerte prematura ${ }^{24}$.
Agradecimientos: Se agradece de manera especial a todos los participantes de la ENS 2009-10, al equipo profesional del Departamento de Salud Pública, de la Facultad de Medicina de la Pontificia Universidad Católica de Chile, quienes desarrollaron y aplicaron la Encuesta Nacional de Salud y al Ministerio de Salud del Gobierno de Chile. Todos los autores revisaron críticamente el manuscrito y están de acuerdo con su versión final. Ninguno de los autores reporta tener conflictos de intereses.

\section{Referencias}

1. Liu PJ, Ma F, Lou HP, Zhu YN. Comparison of the ability to identify cardiometabolic risk factors between two new body indices and waist-to-height ratio among Chinese adults with normal BMI and waist circumference. Public Health Nutr. 2016:1-8. doi: 10.1017/ S1368980016003281.

2. Mozaffarian D, Benjamin EJ, Go AS, Arnett DK, Blaha MJ, Cushman M, et al. Executive Summary: Heart Disease and Stroke Statistics--2016 Update: A Report From the American Heart Association. Circulation. 2016; 133 (4): 447-54. doi: 10.1161/cir.0000000000000366.

3. Arroyo-Johnson C, Mincey KD. Obesity Epidemiology Worldwide. Gastroenterol Clin North Am. 2016; 45 (4): 571-9. doi: 10.1016/j.gtc.2016.07.012.

4. WHO. Obesity: preventing and managing the global epidemic. Report of a WHO consultation. 2000. 0512-3054. Available: http://apps.who.int/iris/bitstream/10665/42330/1/WHO_TRS_894.pdf.

5. MINSAL. Encuesta Nacional de Salud 2009-2010. Chile: Ministerio de Salud, 2010. Available: http:// web.minsal.cl/portal/url/item/bcb03d7bc28b64dfe040010165012d23.pdf.

6. OMS/OPS. Panorama de la Seguridad Alimentaria y Nutricional en América Latina y el Caribe. Santiago, Chile: Organización de las Naciones Unidas para la Alimentación y la Agricultura, Organización Panamericana de la Salud, 2016. Enero 2017. Report No 1. Available: http://iris.paho.org/xmlui/handle/123456789/33680.

7. Alberti KG, Eckel RH, Grundy SM, Zimmet PZ, Cleeman JI, Donato KA, et al. Harmonizing the metabolic syndrome: a joint interim statement of the International Diabetes Federation Task Force on Epidemiology and Prevention; National Heart, Lung, and Blood Institute; American Heart Association; World Heart Federation; International Atherosclerosis Society; and International Association for the Study of Obesity. Circulation. 2009; 120 (16): 1640-5. doi: 10.1161/circulationaha.109.192644. 
8. Dhalwani NN, Zaccardi F, O'Donovan G, Carter P, Hamer M, Yates T, et al. Association Between Lifestyle Factors and the Incidence of Multimorbidity in an Older English Population. The journals of gerontology Series A, Biological sciences and medical sciences. 2016. doi: 10.1093/gerona/glw146.

9. López-Sobaler AM, Rodríguez-Rodríguez E, Aranceta-Bartrina J, Gil A, González-Gross M, Serra-Majem L, et al. General and Abdominal Obesity Is Related to Physical Activity, Smoking and Sleeping Behaviours and Mediated by the Educational Level: Findings from the ANIBES Study in Spain. PloS one 2016; 11 (12): e0169027. doi: 10.1371/journal.pone.0169027.

10. Pengpid S, Peltzer K. Associations between behavioural risk factors and overweight and obesity among adults in population-based samples from 31 countries. Obes Res Clin Pract. 2016. doi: 10.1016/j.orcp.2016.08.001.

11. Bann D, Johnson W, Li L, Kuh D, Hardy R. Socioeconomic Inequalities in Body Mass Index across Adulthood: Coordinated Analyses of Individual Participant Data from Three British Birth Cohort Studies Initiated in 1946, 1958 and 1970. PLoS Med 2017; 14 (1): e1002214. doi: 10.1371/journal.pmed.1002214.

12. WHO. Global Physical Activity Questionnaire: GPAQ version 2.0. World Health Organization, 2009. 2009. Report No. Available: http://www.who.int/chp/steps/ resources/GPAQ_Analysis_Guide.pdf.

13. Bull FC, Maslin TS, Armstrong T. Global Physical Activity Questionnaire (GPAQ): Nine Country Reliability and Validity Study. Journal of Physical Activity \& Health 2009; 6 (6): 790-804. doi:

14. Hoos T, Espinoza N, Marshall S, Arredondo EM. Validity of the Global Physical Activity Questionnaire (GPAQ) in Adult Latinas. Journal of Physical Activity \& Health 2012; 9 (5): 698-705. doi:

15. Ainsworth BE, Haskell WL, Herrmann SD, Meckes N, Bassett DR Jr, Tudor-Locke C, et al. 2011 Compendium of Physical Activities: A Second Update of Codes and MET Values. Medicine and Science in Sports and Exercise 2011; 43 (8): 1575-81. doi: 10.1249/MSS. 0b013e31821ece12.

16. WHO. Global recommendations on physical activity for health. World Health Organization, 2010. Available: http://www.who.int/dietphysicalactivity/publications/9789241599979/en/

17. Dussaillant C, Echeverría G, Villarroel L, Marín PP, Rigotti A. Unhealthy food intake is linked to higher prevalence of metabolic syndrome in Chilean adult population: cross sectional study in 2009-2010 National Health Survey. Nutricion Hospitalaria 2015; 32 (5): 2098-104. doi: 10.3305/nh.2015.32.5.9657.

18. Ortiz H, Galan I, Zorrilla B, Martínez-Cortés M. [Factors associated with overweight, general obesity and abdominal obesity in the Madrid Community's adult population. Study PREDIMERC]. Med Clin (Barc) 2014; 143 (12): 560-2. doi: 10.1016/j.medcli.2013.09.025.

19. Lanas F, Bazzano L, Rubinstein A, Calandrelli M, Chen CS, Elorriaga N, et al. Prevalence, Distributions and Determinants of Obesity and Central Obesity in the Southern Cone of America. PloS one 2016; 11 (10): e0163727. doi: 10.1371/journal.pone.0163727

20. Trivedi T, Liu J, Probst J, Merchant A, Jhones S, Martin $\mathrm{AB}$. Obesity and obesity-related behaviors among rural and urban adults in the USA. Rural and remote health 2015; 15 (4): 3267. doi:

21. Klesges RC, Meyers AW, Klesges LM, Lavasque ME. Smoking, body-weight, and their effects on smoking-behavior-a comprehensive review of the literature. Psychological Bulletin 1989; 106 (2): 204-30. doi: 10.1037//0033-2909.106.2.204.

22. Monasta L, Batty GD, Cattaneo A, Lutje V, Ronfani L, van Lenthe FJ, et al. Early-life determinants of overweight and obesity: a review of systematic reviews. Obesity Reviews 2010; 11 (10): 695-708. doi: 10.1111/j.1467789X.2010.00735.x.

23. Hardikar S, Hochenberger R, Villringer A, Ohla K. Higher sensitivity to sweet and salty taste in obese compared to lean individuals. Appetite 2016; 111: 158-65. doi: 10.1016/j.appet.2016.12.017.

24. WHO. Global action plan for the prevention and control of noncommunicable diseases 2013-2020. Geneva, Switzerland: World Health Organization, 2013. Available: http://apps.who.int/iris/bitstream/10665/ 94384/1/9789241506236_eng.pdf. 